\title{
Cognitive Radio Network with Enhanced Protocol for Maximum Throughputs
}

\author{
Ms. Shubhangi Mahamuni ${ }^{1}$, Dr. Vivekanand Mishra ${ }^{2}$, Dr. Angela Amphawan ${ }^{3}$ \\ ${ }^{\prime}$ (E\&TC Department, MIT's Maharashtra Academy of Engineering, India) \\ ${ }^{2}$ (Electronics Department, SVNIT, India) \\ ${ }^{3}$ (InterNetWorks Research Laboratory, School of Computing, Universiti Utara Malaysia, Kedah, Malaysia)
}

\begin{abstract}
In this paper, a Cognitive Radio based Medium Access Control (CR-MAC) protocol for wireless sensor networks that utilizes cognitive radio transmission is used. In cognitive radio (CR) networks, identifying the available spectrum resource through spectrum sensing, deciding on the optimal sensing and transmission times, and coordinating with the other users for spectrum access are the important functions of the medium access control (MAC) protocols. In this paper, the sensor nodes are classified into nodes of critical information, and nodes of non-critical information. The CR-MAC protocol prioritizes the critical packets access to the transmission medium by transmitting them with higher power while transmitting lower priority packets using lower transmission power. The network throughputs can be improved by increasing number of traffic rate, also the end-to-end delay will minimizes by CR-MAC protocol. And a motive to take advantage of heavy traffic rate which may occur at the receiver, a higher priority packet experience collision only when there are more than one critical packet transmission at the same time slot while non critical packets experience collision when there are more than one transmission at the same time slot.
\end{abstract}

Keywords - Cognitive radio, Wireless sensor network, CR-MAC

\section{Introduction}

Accordingly today's trend of technology the demand for wireless communication introduces efficient spectrum utilization challenge. To complete this challenge, cognitive radio has emerged as the key technology, which enables opportunistic access to the spectrum. The main potential advantages introduced by cognitive radio are improving spectrum utilization and increasing communication quality. These appealing features match the unique requirements and challenges of resource-constrained multi-hop wireless sensor networks (WSN). (1) Cognitive radio is an emerging wireless communications concept in which a network or a wireless node is able to sense its environment, and especially spectrum holes, and change its transmission and reception chains to communicate in an opportunistic manner, without interfering with licensed users. Cognitive radio thus aims to improve the way the scarce radio spectrum is utilized [2][3].The importance of differentiating traffic in wireless sensor networks is growing, and guaranteeing different QoS levels is considered a key challenge for research on wireless sensor networks [4][5].The former approach is mainly focused on infrastructure based networks, in which a centralized coordinator or base station manages the spectrum allocation and sharing among the CR users. The CR users, however, may participate in the spectrum sensing function and provide channel information to the central controller. The standardization efforts lead to uniformity in design and policy, thereby allowing multiple independent CR operators to coexist. [6,7] As an example, the carrier sense mechanism at the MAC layer may not reveal complete information regarding the channel owing to its inability to distinguish between the energy radiated by other CR users and the active PUs in the spectrum. [8] In addition, packets may be simply retransmitted in the event of a collision with other CR users, while the transmission must cease immediately if the packet loss is due to PU activity. [9]

The geographic greedy forwarding protocol that we are going to implement works as follow.

- On receiving a packet from upper layer, the source node adds a header including the destination ID and location.

- The source node sends out the packet to the neighbor closest to the destination.

- On receiving a packet from a neighbor, a node forwards the packet if there exists a neighbour closer to the destination than itself.

- If there is no neighbor closer than itself, so called local minimum, it simply drops the packet.

In the analysis of CR MAC protocol, we first give the following definitions:

- $N_{c}$ :sensor nodes of critical information

- $N_{n e}$ :sensor nodes of non critical information 
- $A_{c}$ :aggregate critical packet arrival rate per time slot

- $A_{m \varepsilon}:$ aggregate non critical packet arrival rate per timeslot

- $T_{c}$ : critical traffic throughput

- $T_{n e}$ : non critical traffic throughput

- $D_{c}$ : critical traffic rejection rate

- $D_{n e}$ : non critical traffic rejection rate

- $\lambda c$ c critical packet arrival rate

- $\lambda$ nc: non critical packet arrival rate

- $r_{c}$ : number of allowed packet retransmissions for critical nodes

- $r_{n e}$ :number of allowed packet retransmissions for non-critical nodes

In the analysis of the critical traffic throughput as its transmission is independent of non critical traffic transmission. Then, the obtained results are integrated within the analysis of the non critical traffic throughput which depends on the critical traffic transmission only

\subsection{Critical Traffic Throughput Analysis:}

Generally, the total offered critical traffic, Ac, ready for transmission from the beginning of a time slot can be defined as follows:

$$
\begin{gathered}
A_{c}=\lambda_{c}+\lambda_{c}(1-P c)+\lambda_{c}(1-P c)^{2}+\ldots . . \\
\ldots \ldots \ldots+\lambda_{c}(1-P c)^{r_{c}} \\
=\lambda_{c} \sum_{k=0}^{r_{c}}(1-P c)^{r_{c}} \\
=\lambda_{c} \frac{1-\left(1-P_{c}\right)^{r_{c}+1}}{P_{c}}
\end{gathered}
$$

The parameter Pc in Equation (1) is the probability of a successful critical packet transmission in a time slot. The general expression of this parameter is defined in the right hand side of Equation (2).

$$
P_{C}=\left[1-\frac{A_{C}}{N_{C}}\right]^{N_{C}-1}
$$

After substituting the parameter Pc, Equation (1) can be rewritten as in Equation (3).

$$
A_{C}=\lambda_{c} \frac{1-\left[1-\left(1-\frac{A_{C}}{N_{C}}\right)^{N_{C-1}}\right]^{T_{C}+1}}{\left(1-\frac{A_{C}}{N_{C}}\right)^{N_{C-1}}}
$$

The optimal critical packet throughput, Sc, in a time slot is obtained by multiplying the critical packet successful transmission probability, pc, by the aggregate critical traffic, Ac. Therefore, Equation (3) can be rewritten as in Equation (4).

$$
A_{c}=\lambda_{c}\left[1-\left\{1-\left(1-\frac{A_{C}}{N_{C}}\right)^{N_{C}-1}\right\}^{r_{c}+1}\right]
$$

From Equation (4), it can be said that the critical traffic throughput is independent of the non critical packet transmission as there are only critical node terms. This is due to the use of cognitive radio transmission where critical packets transmitted using higher power while non critical packets are transmitted with lower 
power. Therefore, the receiver will consider the received lower power of non critical traffic as a noise when it coincides with a single critical packet received power.

\subsection{Non-Critical Traffic Throughput Analysis:}

Whereas in this non critical transmission, the successful non critical packet transmission depends on both critical and non critical traffic arrival per time slot. Therefore, the successful non critical packet transmission in a time slot occurs only when there is a single non critical packet arrival and zero critical packet arrival. These conditions are stated in the equation of the probability of successful non critical packet transmission Pnc (Equation (5)).

$$
P_{n c}=\left(1-\frac{A_{n c}}{N_{n e}}\right)^{N_{n e}-1}\left(1-\frac{A_{C}}{N_{C}}\right)^{N_{C}}
$$

The derivation of the aggregate non critical packet traffic arrival, Anc, per time slot, is as follows:

$$
\begin{gathered}
A_{n c}=\lambda_{n c}+\lambda_{n c}(1-P n c)+\lambda_{n c}(1-P n c)^{2}+\ldots \ldots \ldots+\lambda_{n c}(1-P n c)^{r_{n c}} \\
=\lambda_{n c} \sum_{k=0}^{r_{n c}}(1-P n c)^{r_{n c}} \\
=\lambda_{n c} \frac{1-\left(1-P_{n c}\right)^{r_{n e}+1}}{P_{n c}}
\end{gathered}
$$

After substituting the term pnc with the right-hand side of Equation (5), Equation (6) can be simplified as below:

$$
A_{n c}=\lambda_{n c} \frac{1-\left[1-\left(1-\frac{A_{n c}}{N_{n c}}\right)^{N_{n c}-1}\left(1-\frac{A_{c}}{N_{c}}\right)^{N_{c}}\right]^{r_{n e}+1}}{\left(1-\frac{A_{n c}}{N_{n e}}\right)^{N_{n c}-1}\left(1-\frac{A_{c}}{N_{c}}\right)^{N_{c}}}
$$

The non critical packet throughput, Snc, is obtained by multiplying Equation (7) by the probability of successful transmission for the non critical packet traffic. The general formula for the non critical traffic throughput, Snc, is presented in Equation (8).

$$
T_{n c}=A_{n c}\left(1-\frac{A_{n c}}{N_{n c}}\right)^{N_{n c}-1}\left(1-\frac{A_{c}}{N_{c}}\right)^{N_{c}}
$$

\section{Network Traffic Rejection Analysis}

The important performance parameter studied in this paper is the critical and non critical packet traffic rejection rate. The derivation of the critical and non critical packet traffic rejection formulas are presented herein. The critical traffic rejection probability depends on the number of allowed critical packet retransmissions. In the case of first packet transmission, the probability of packet transmission failure is $(1-P n c)$. the probability of critical packet transmission failure given $r_{c}$ allowed retransmission is $(1-P n c)^{r_{n e}+1}$. Therefore, their general formula for critical traffic rejection rate for $r_{c}$ allowed packet retransmissions can be written as in Equation (9).

$D_{C}=\left[1-\left(1-\frac{A_{C}}{N_{C}}\right)^{N_{n e}-1}\right]^{r_{C}+1}$ 
Similarly, the non critical traffic rejection probability in the case of first non critical packet transmission is.

When there $r_{n e}$ are allowed non critical packet retransmissions; this rejection probability is defined in (10).

$D_{n e}=\left[1-\left(1-\frac{A_{n c}}{N_{n e}}\right)^{N_{n c}-1}\left(1-\frac{A_{c}}{N_{n}}\right)^{N_{c}-1}\right]^{\gamma_{c}+1}$

The critical and critical traffic rejection rate formulas are also solved numerically to compute the system rejection rate under different network parameters settings.

\section{FIGURES AND TABLES}

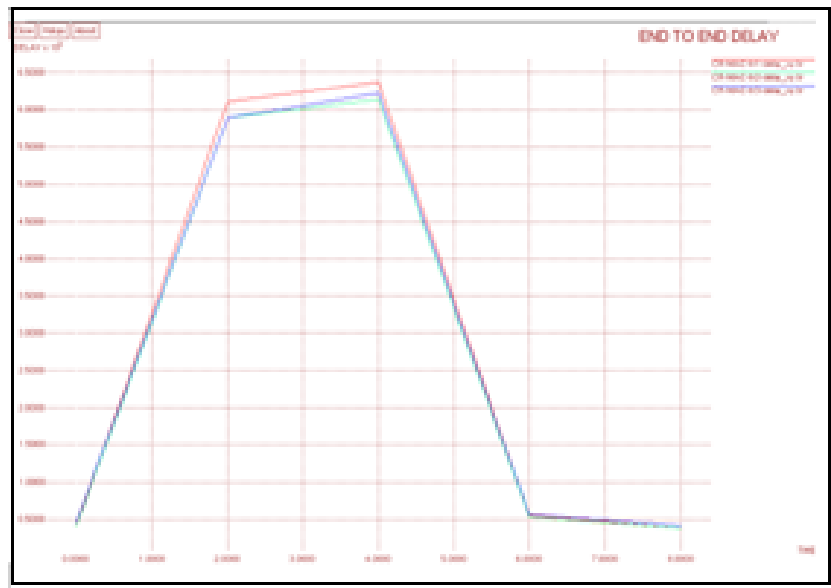

Fig.1: End to End Delay in different traffic

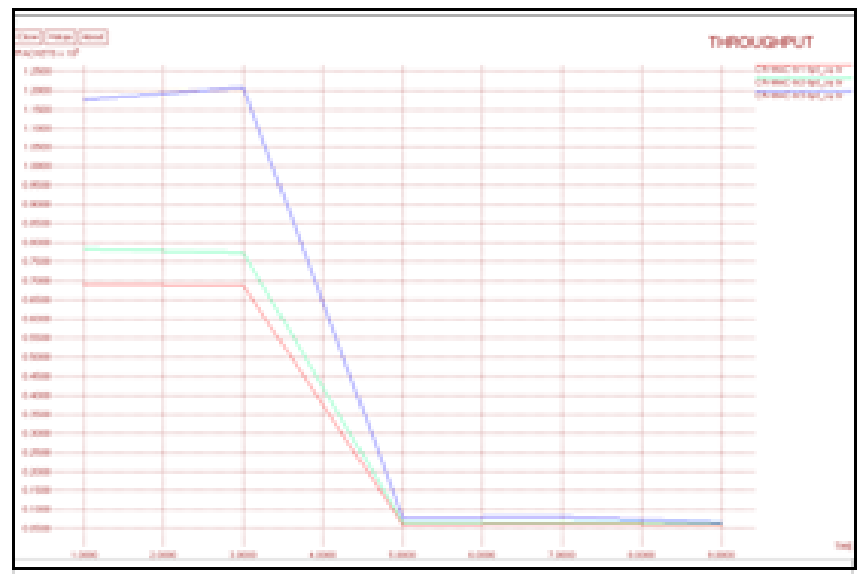

Fig.2: Throughputs at Different traffic

\section{CONCLUSION}

Thus from the mathematical and simulation analysis We have been calculated end to end delay and throughput at different traffic. The end to end delay is less as compared to conventional system and throughput is more as compared to conventional system.

\section{Acknowledgements}

We hereby take this opportunity to express profound thanks and gratitude from the bottom of my hearts towards SVNIT, Surat and MITCOE for providing valuable platform and untiring encouragement during the preparation of this paper. 


\section{Journal Papers:}

\section{REFERENCES}

[1] M Ozaki, Y. Adachi, Y. Iwahori, and N. Ishii, Application of fuzzy theory to writer recognition of Chinese characters, International Journal of Modelling and Simulation, 18(2), 1998, 112-116.

[2] Ozgur B. Akan Osman B. Karli Ozgur, Ergul Next generation Wireless Communications Laboratory (NWCL) E-mail, J. Mitola, "Software radios: Survey, critical evaluation and future directions",IEEE Aereosp. Electro.Syst. Mag., Vol. 8, pp. 25-36, Apr. 1993.

[3] R. Rubenstein, "Radios get smart", IEEE Spectrum, Feb. 2007. Bucaille, A. Tonnerre, L. Ouvry, and B. Denis. "MAC layer design for UWB LDR systems: PULSERS proposal". 4th Worksshop on Positioning, Navigation and Communication 2007 (WPNC'07, pages 277 \{2837, March 2007.

[4] J. Mitola Iii And G. Q. Maguire, Jr."Cognitive Radio: Making Software Radios More Personal". IEEE Personal communications, 6(4):13\{18, August 1999.

[5] J. Mitola Iii And G. Q. Maguire, Jr. "Cognitive Radio: Making Software Radios More Personal". IEEE Personal Communications, 6(4):13-18, August 1999.

[6] N. Timmons, and W. Scanlon, "Analysis of the Performance of IEEE 802.15 .4 for Medical Sensor Body Area Networking". IEEE Conference on Sensor and Ad Hoc Communications and Networks, pages 16 - 24, October 2004.

[7] S. Haykin "Cognitive Radio: Brain-Empowered Wireless Communications". IEEE Journal On Selected Areas in Communications, 23(2):201 - 220, February 2005.

[8] T. Watteyne, I. Augt'e-Blum, and S. Ubteda. "Dual-mode real-time mac protocol for wireless sensor networks: a validation/simulation approach”, International Conference on Integrated Internet Ad Hoc and Sensor Networks, 138(2), May 2006,theory and Practice of Geographic Routing Stefan Ruhr up “Department of Computer Science University of Freiburg, Germany February 2009. 